\title{
Children with Complex Medical Conditions: an Under-Recognized Driver of the Pediatric Cost Crisis
}

\section{Nancy A. Murphy, MD FAAP FAAPMR ${ }^{1,2, *}$ Edward B. Clark, MD FAAP}

\author{
Address \\ ${ }^{1}$ Department of Pediatrics, University of Utah School of Medicine, Salt Lake City, \\ USA \\ ${ }^{*}, 2$ Division of Pediatric Physical Medicine \& Rehabilitation, 81 Mario Capecchi \\ Drive, Salt Lake City, UT, 84113, USA \\ Email: Nancy.murphy@hsc.utah.edu
}

Published online: 7 0ctober 2016

(C) Springer International Publishing AG 2016

This article is part of the Topical Collection on Quality Improvement

Keywords Children with medical complexity $\cdot$ Comprehensive care $\cdot$ Value proposition $\cdot$ Pediatric healthcare costs Palliative care

\section{Opinion statement}

Children with medical complexity (CMC) represent less than $1 \%$ of all US children, but account for more than $30 \%$ of total pediatric healthcare costs. They are a rapidly growing population of children with the highest levels of medical fragility and intensive healthcare needs that drive them in and out of inpatient settings, particularly intensive care units, at astounding frequencies. CMC account for $34 \%$ (\$1.6 billion) of all Medicaid pediatric healthcare expenditures, $47 \%$ of Medicaid's total spending on pediatric hospital care, and for $71 \%$ of the 30 -day unplanned hospital readmissions. As major drivers of the national pediatric healthcare cost crisis, CMC urgently need high value systems of care that are responsive to their longitudinal needs. We encourage healthcare leaders to recognize the unique needs of CMC and their families and to provide intensive inpatient and outpatient comprehensive care in team-based models of goal-directed care. We emphasize the importance of safety, of rapid access to home and community based care, and of continuity of care across acute inpatient, post-acute, and outpatient settings. Resources of palliative care, post-acute care, telehealth, and strong parent-provider partnerships are needed to support value-driven systems. Emerging evidence suggests that comprehensive care for CMC and their families drives total healthcare expenditures down and offers an innovative approach to the provision of high value care for this growing population of children. Efforts to design, implement, and measure outcomes for innovative systems of care for CMC are needed. 


\section{Introduction}

The US healthcare system is in crisis. Rising costs are driven by disproportionate spending on the aging Medicare population, disadvantaged adults on Medicaid, and inefficiencies of our system [1]. Lost in the clamor of this conversation is the plight of a small group of children and their families with complex medical conditions. They are among the highest utilizers of healthcare resources and are on a trajectory that likely will extend from childhood through decades of adult life, accruing lifetime costs well beyond anything imaged for an older adult population [1]. In this paper, we explore the origins of the pediatric healthcare crisis, describe the emerging social and financial consequences, review some early efforts to deliver comprehensive care for children with medical complexity and their families, and provide a framework for the development and implementation of high value systems of care.

\section{A growing pediatric population}

While the vast majority of children are healthy, children with special health care needs account for $15 \%$ of the US pediatric population [2]. Within this group are a much smaller and yet rapidly growing group of children with medical complexity (CMC), who have the highest degree of medical fragility and the most intensive healthcare needs [3]. Although CMC comprise only 0.4 to $0.7 \%$ of all US children ( $320,000-560,000$ children), they account for $15-33 \%$ of all pediatric healthcare costs ( $\$ 50-\$ 110$ billion per year) [4]. They are frequently graduates of neonatal intensive care and surviving with previously lethal conditions including extreme prematurity, perinatal conditions, congenital anomalies, chronic lung disease, and neurologic impairments. They depend upon an array of invasive procedures, innovative technologies, and multidisciplinary teams to address their needs and keep them well.

\section{Technology dependent and Medicaid funded}

Many CMC have technology dependencies and high levels of medical fragility that drive them in and out of inpatient settings, particularly intensive care units at astounding frequencies. Most $\mathrm{CMC}$ are covered by Medicaid, accounting for $34 \%$ ( $\$ 1.6$ billion) of all Medicaid pediatric healthcare expenditures. There is a high concentration of need, as $5 \%$ of these children account for $50 \%$ of the total spend [4]. CMC account for $47 \%$ of Medicaid's spending on hospital care for all children, and for $71 \%$ of the 30-day unplanned hospital readmissions $[5,6]$.

\section{Shift in hospitalization to children with complex conditions}

Over the last two decades, children's hospitals have shifted from caring for acutely ill children to the inpatient management of those with complex medical conditions and fragile health status. In 1997, children with one or more chronic conditions accounted for only $9 \%$ of pediatric inpatient admissions, $23 \%$ of hospital days, and $37 \%$ of total charges [7]. In 2006, barely a decade later, CMC accounted for $42 \%$ of all US pediatric hospitalizations [8], and children with neurologic impairments accounted for nearly $30 \%$ of hospital charges that same year [6]. Between 2004 and 2009, hospitalizations for children with chronic conditions affecting two or more body systems increased by $32 \%$, and accounted for $19.2 \%$ of pediatric admissions; cerebral palsy and asthma were the most common primary diagnoses in this group $[9 \bullet \bullet]$. Among the children who accounted for the highest annual inpatient expenditures in 2010, the top $10 \%$ most expensive children constituted $56.9 \%$

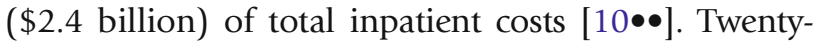
seven percent of these children experienced persistently high costs, particularly those with chronic respiratory conditions and technology dependencies. These trends demonstrate the high and rapidly growing inpatient resource utilization of $\mathrm{CMC}$.

We believe that many hospitalizations for CMC are driven by a lack of appropriate, responsive, and accessible community-based services. Without rapid access to outpatient providers who are familiar with a child's individualized needs and family resources, families opt for emergency department (ED) visits. Overwhelmed by time pressures and the complexity of care planning, emergency physicians often choose to admit $\mathrm{CMC}$ and rely on inpatient teams to sort out the acute and chronic aspects of their conditions. The result is an escalation of care and costs, with uncertain outcomes.

\section{Out of hospital complex care}

$\mathrm{CMC}$ are not only high utilizers of inpatient care but are also of healthcare resources across the continuum. Analysis of a population-based sample of CMC in Ontario, Canada, from 2005 to 2007 demonstrated that CMC accounted for one third of child health spending, particularly related to hospital readmissions ( $27 \%$ of costs) 
but also related to home care (11\% of costs) and physician services ( $6 \%$ of costs) [11]. CMC have an annual mean of 19 outpatient visits, high home care needs, and disproportionate needs for costly prescription medications. In a US tertiary pediatric center, $20 \%$ of the emergency room visits were for children with chronic conditions [12]. Yet, despite this large investment of resources, families of $\mathrm{CMC}$ report lower satisfaction with health care [13].

\section{Defining a value proposition for the child with medical complexity}

Healthcare transformation has a key focus on the value proposition, high quality care at the lowest appropriate cost. Yet value remains largely unmeasured and misunderstood [14]. The value proposition for children with medical complexity is far more difficult because summing the costs of hospitalizations, diagnostic tests, medications, home health care, and durable medical equipment is neither adequate nor sufficient. The total value equation must also consider direct nonmedical costs (out of pocket expenses, costs of travel to hospital) and indirect consequences (lost productivity for economy) [15]. Achieving high value for pediatric patients must become the overarching goal of healthcare delivery. Rigorous, disciplined measurement and improvement of value are our best longitudinal solution to the national healthcare cost crisis.

\section{Financial stress to families}

The social costs of care are a significant component of expense and stress for families. Over half (57\%) of parents of CMC in the 2005-2006 National Survey of CSHCNs report financial problems, that a family member stopped working because of the child's health (54\%), and at least one unmet medical service need (49\%); $33 \%$ report difficulty in accessing nonmedical services [16]. When surveyed, $68 \%$ of families of CMC reported financial hardship, and $46 \%$ reported social hardship [17•]. These hardships might further drive total costs of care, as overwhelmed and depleted families struggle to meet the complex medical and functional needs of their CMC while also meeting competing family and personal responsibilities [18].

\section{Social determinants: when family care fails, what is the safety net?}

When the needs of CMC exceed the resources, abilities, and endurance of their parents to meet them, families face challenging decisions. Although medical foster care can be an option for sustaining in-home care, foster parents report inadequate training and preparation to care for children with extremes of medical and social risk, and shared decision making among foster families, caseworkers, biologic families, legal advocates, and clinicians can further undermine care [19]. Decisions regarding out-of-home placements for $\mathrm{CMC}$ can be stressful for families and are fraught with uncertainty regarding value, as outcomes and costs for individuals, families, and societies are highly variable and poorly quantified [20]. Hospitalizations can be prolonged when discharge dispositions are uncertain.

Post-acute care continues to be an underutilized care option for $\mathrm{CMC}$ who might benefit from weeks to months of sustained intensity, daily caregiving throughout a period of recovery in a less restrictive and less costly environment [21]. Among all pediatric discharges in 2012, $5 \%$ were to home health care ( $44 \%$ for neonatal care), and only $1 \%$ to post-acute care ( $43 \%$ had nonneonatal respiratory, musculoskeletal, and traumarelated conditions) $[22 \bullet \bullet]$.

When families face difficult decisions regarding medical foster care, post-acute care, out of home placements, and longitudinal goals of care for their children, pediatric palliative care programs can offer much needed support. Palliative care lowers costs among patients approaching death and increases the value of lifelong health care among this population of high-resource utilizers [23].

\section{Variability of care}

Depending on where they receive care, CMC have widely different patterns of utilization which reflect an overall lack of comprehensive care plans, explicitly defined provider and parent goals of care, and availability and implementation of evidence-based approaches in highquality acute care hospitals, pediatric skilled nursing facilities, and long-term acute care units. Wide variations are seen nationally in the use of home health care and post-acute care settings (rehabilitation, long-term acute care, and skilled nursing units) for $\mathrm{CMC}$ upon discharge from acute hospital units, with variations influenced by

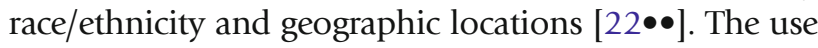
of electrocardiography and brain MRIs for inpatient CMC also varies widely [24], as does the use of inhaled nitric oxide for premature infants [25]. Wide variations in care are inherently related to variations in value, affecting both costs and outcomes.

The dynamic interplay of acute and chronic conditions in CMC challenges all aspects of care and treatment. A recent study of hospitalized children with 
neurologic impairments and pneumonia at 40 US children's hospitals demonstrated that children with aspiration pneumonia have longer lengths of stay, more ICU transfers, greater hospitalization costs, and more 30-day readmissions ( 17.4 vs $6.8 \%$ ) than children with nonaspiration related pneumonia [26]. Although providers might be inclined to group all hospitalized children with pneumonia into a single group, it is important to recognize that differences in etiology and underlying conditions most certainly affect outcomes.

\section{Vulnerable in the system}

Medical errors are a threat to all hospitalized patients, but most especially to those with chronic, complex medical conditions. CMC typically require complex medication schedules, compounded formulations, enteral feedings, and technology supports. They depend on their family to accurately articulate detailed care plans and to describe acute changes in clinical status, often when challenged by stress and fatigue. Risk of errors in such scenarios is significant. Additionally, healthcare providers who may not be familiar with the child or family are at increased risk for under or over estimating acute changes superimposed on chronic conditions. Investment of time-intensive care is essential to ensure the provision of safe care for CMC in healthcare settings. Although time is a universally limited resource, it is a key driver of value.

\section{A way forward: comprehensive care}

The complex medical and functional needs of CMC are lifelong and are best addressed by teams that provide value-driven care based on continuously updated longitudinal care plans that promote the best interests of the child and goals of their families. But, who best provides the care? Where is comprehensive care best be rendered? How is it paid for? We offer our thoughts on the Who, the Where, and the How.

\section{The Who}

Sixty-five percent of primary care pediatricians feel that primary care is the optimal setting for medical homes for most CSHCN, while $43 \%$ agree that subspecialty care is the best setting for the care of $\mathrm{CMC}$ [27]. Cost and time are cited as the leading barriers to the implementation of primary care medical homes, as well as skills in the communication and coordination of complex care. The Who is intertwined with the Where and the How.

\section{The Where}

Community-based, primary care pediatric medical homes often struggle to be the hub of comprehensive care for $\mathrm{CMC}$, not because of professional competency but rather based on the unique and individualized needs of this population. Community clinics expertly deliver high volumes of problem-focused, acute, and episodic care. Yet CMC are, by definition, medically, functionally, and psychosocially complex and fragile, and their care demands high investments of time, clinical intuition, and deliberate communication with providers and families. Therefore, hospital-based comprehensive care programs seem to be a promising option for the delivery of high value health care for $\mathrm{CMC}$. These programs must be tailored to the demography, geography, and community. One size does not fit all.

We share three examples of comprehensive care programs for CMC. These programs vary in their scope of services, primary versus consultative models of care, and geographic locations. Although it is difficult to extract generalizable principles from current experiences beyond that of the CMC and their families, we must ask, at what cost do these clinical and family outcomes come?

- Gordon showed that a consultative, tertiary hospital-based consultation program for CMC was associated with decreased hospital days, increased clinic visits, and a reduction in tertiary care center charges of $\$ 10.7$ million for 227 children [28].

- Casey's rural children's hospital-based multidisciplinary primary care clinic for $\mathrm{CMC}$ demonstrated decreased costs for inpatient and ED care, with overall Medicaid cost reductions of $\$ 1180$ per child per month [29].

- In a randomized study of comprehensive care versus usual pediatric primary care, Mosquera found that the comprehensive care model was associated with reductions in ED visits, frequency and length of hospitalizations, pediatric ICU admissions, and total costs; Medicaid payments were reduced by

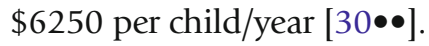

Families of CMC enrolled in these tertiary care center-based structured clinical programs describe increased satisfaction with access to primary care checkups, therapies, mental health care, respite care, and referrals [31]. 


\section{The How}

Comprehensive care cannot survive in a fee for service system. The cost of a comprehensive care team cannot be met on an RVU schedule because phone calls, coordination of services with multiple agencies, care planning among providers and families, and discussions with payers are not currently reimbursable. We believe that, as prospective payment models emerge, hospital-based comprehensive care programs for $\mathrm{CMC}$ offer the best healthcare value, as do community-based pediatric medical homes for typically developing children and those with non-complex special healthcare needs.

\section{Our experience}

At the University of Utah and Primary Children's Hospital, we have over 8 years of experience in delivering pediatric comprehensive care in a consultative model, collaborating closely with community providers. Our Comprehensive Care Program is staffed by two pediatric physiatrists, a pediatric generalist, two midlevel providers, and two care managers, with additional supports from social workers, dieticians, and medical assistants in an integrated system of care. The program is financed by the Department of Pediatrics. In our assessment, the value is in the avoidance of high-cost hospitalizations and unnecessary visits to emergency departments. Additionally, when hospitalization is necessary, comanagement with hospitalist teams supports value. We have seen cost avoidance of $\$ 116$ million for a cohort of 568 children with medical complexity over 7 years, including $\$ 69$ million for 80 children in our Trach-Vent program over the same time interval. Overall, we estimate a cost avoidance of more than $\$ 15$ million per year for this population of CMC.

\section{Building the culture and teams for comprehensive care} It is imperative that the current and future pediatric workforce is well prepared to meet the multifaceted healthcare needs of increasing populations of CMC. However, training opportunities can be limited. For example, $\mathrm{CMC}$ in one major tertiary pediatric hospital had $29 \%$ lower costs and $38 \%$ fewer hospital days when admitted to staff hospitalists rather than subspecialist and/or house staff teams, yet this limits the development of future pediatricians [32]. Residents and young physicians at a US tertiary-care medical center identified a lack of effective training as one of the major challenges in caring for $\mathrm{CMC}$, and identified the need for more longitudinal patient relationships into their residency experiences [33•]. Future pediatricians need education and experience in the care of CMC and their families, and residency programs are challenged to balance the demands of work hour restrictions with meaningful educational experiences in the longitudinal delivery of complex comprehensive care.

\section{Conclusion: next steps}

As more and more systems are taking risk for their populations, it is imperative that leaders remember and consider emerging populations of $\mathrm{CMC}$. We suggest a path to identifying the population, defining their needs and implementing systems for their care.

- Identify a population of $\mathrm{CMC}$ from large payer and hospital administrative data sets, including patterns of hospital utilization (including ER visits), technology dependencies, and total health care costs.

- Understand the needs of CMC and their families, starting with data analyses and then including input from key stakeholders, particularly parents. Remember that small groups of children of the highest medical complexity account for major total healthcare expenditures, and that intensive inpatient and outpatient comprehensive care in teambased models of goal-directed care can have high impact.

- Emphasize a culture of safety and recognize the high risk for error in caring for CMC. When admitted, create alert systems and heightened awareness of each child and his or her individualized needs.

- Implement strategies that predict complexity and intervene early. For example, recognize that preterm birth, neonatal encephalopathy, sepsis, and other conditions of the newborn, and congenital anomalies contribute to 4 of the top 25 top causes of lifetime disability [15]. Anticipatory guidance for families and providers is key.

- Ensure continuity of care, beginning in the NICU or PICU and continuing through hospitalizations, post-acute care, and long-term management. Understand that discharge planning is an anachronism.

- Recognize the role of post-acute care in the provision of cost-effective and safe care for $\mathrm{CMC}$, measure evidence of outcomes, and refine models of care to promote best practices.

- Maintain continuous links with families of CMC through home visits, trusted and predictable care managers, telehealth services that promote rapid 
access to appropriate care, and home-based technologies that reduce ED and inpatient care.

- Deploy home-based urgent care teams. Although seemingly expensive, these long-term satisfiers can short circuit the pursuit of care of higher acuity and higher cost.

- Care for families of CMC, including parents and siblings. Be aware of the stress of chronic illness and disability on children, families, healthcare systems, and societies.

- Engage all stakeholders, including ethicists and palliative care providers, in the longitudinal care of $\mathrm{CMC}$ and their families, particularly when issues of futility and complex decision-making arise.

- Measure value of care systems for CMC, including costs and outcomes at the level of the child, the family, the healthcare system, and the society.

\section{Compliance with Ethics Guidelines}

\section{Conflict of Interest}

Nancy A. Murphy declares that she has no conflict of interest. Edward B. Clark declares that he has no conflict of interest.

Human and Animal Rights and Informed Consent

This article does not contain any studies with human or animal subjects performed by any of the authors.

\section{References and Recommended Reading}

Papers of particular interest, published recently, have been highlighted as:

- Of importance

$\bullet \quad$ Of major importance

1. NHE Fact Sheet. CMS.gov; 2014. https://www.cms. gov/research-statistics-data-and-systems/statisticstrends-and-reports/nationalhealthexpenddata/nhefact-sheet.html. Accessed 4/2/2016.

2. U.S. Department of Health and Human Services, Health Resources and Services Administration, Maternal and Child Health Bureau. The national survey of children with special health care needs chartbook 2009-2010. Rockville: National Center for Health Statistics at the Centers for Disease Control; 2013.

3. Cohen E, Kuo DZ, Agrawal R, et al. Children with medical complexity: an emerging population for clinical and research initiatives. Pediatrics.

2011;127(3):529-38.

4. Berry JG, Hall M, Neff J, et al. Children with medical complexity and Medicaid: spending and cost savings. Health Aff (Millwood). 2014;33(12):2199-206.

5. Berry JG, Hall DE, Kuo DZ, et al. Hospital utilization and characteristics of patients experiencing recurrent readmissions within children's hospitals. JAMA. 2011;305(7):682-90.

6. Berry JG, Poduri A, Bonkowsky JL, et al. Trends in resource utilization by children with neurological impairment in the United States inpatient health care system: a repeat cross-sectional study. PLoS Med. 2012;9(1), e1001158.
7. Simon TD, Berry J, Feudtner C, et al. Children with complex chronic conditions in inpatient hospital settings in the United States. Pediatrics. 2010;126(4):647-55.

8. Miller RL, Gebremariam A, Odetola FO. Pediatric highimpact conditions in the United States: retrospective analysis of hospitalizations and associated resource use. BMC Pediatr. 2012;12:61.

9.• Berry JG, Hall M, Hall DE, et al. Inpatient growth and resource use in 28 children's hospitals: a longitudinal, multi-institutional study. JAMA Pediatr. 2013;167(2):170-7.

In this key publication, Berry uses the Pediatric Health Information System database to identify and describe trends in the inpatient care of children with chronic, complex conditions.

10.• Peltz A, Hall M, Rubin DM, Mandl KD, Neff J, Brittan $\mathrm{M}$, et al. Hospital Utilization Among Children With the Highest Annual Inpatient Cost. Pediatrics. 2016;137(2):e20151829.

This retrospective study describes the healthcare costs and utilization of the children who account for the top $10 \%$ of total inpatient costs, and complements studies that have examined costs according to chronic conditions of childhood.

11. Cohen E, Berry JG, Camacho X, Anderson G, Wodchis W, Guttmann A. Patterns and costs of health care use of 
children with medical complexity. Pediatrics.

2012;130(6):e1463-70.

12. O'Mahony L, O'Mahony DS, Simon TD, Neff J, Klein EJ, Quan L. Medical complexity and pediatric emergency department and inpatient utilization. Pediatrics. 2013;131(2):e559-65.

13. Kuo DZ, Melguizo-Castro M, Goudie A, Nick TG, Robbins JM, Casey PH. Variation in child health care utilization by medical complexity. Matern Child Health J. 2015;19(1):40-8.

14. Porter ME. What is value in health care? N Engl J Med. 2010;363(26):2477-81.

15. Dukhovny D, Pursley DM, Kirpalani HM, Horbar JH, Zupancic JA. Evidence, quality, and waste: solving the value equation in neonatology. Pediatrics. 2016;137(3):1-10.

16. Kuo DZ, Cohen E, Agrawal R, Berry JG, Casey PH. A national profile of caregiver challenges among more medically complex children with special health care needs. Arch Pediatr Adolesc Med. 2011;165(11):1020-6.

17. Thomson J, Shah SS, Simmons JM, Sauers-Ford HS, Brunswick S, Hall D, et al. Financial and Social Hardships in Families of Children with Medical Complexity. J Pediatr. 2016;172:187-93 e1.

These authors describe the hardships of families of $\mathrm{CMC}$, using survey methodology and a comparison group of families of children with asthma.

18. Murphy N, Christian B. Disability in children and young adults: the unintended consequences. Arch Pediatr Adolesc Med. 2007;161(10):930-2.

19. Seltzer RR, Henderson CM, Boss RD. Medical foster care: what happens when children with medical complexity cannot be cared for by their families? Pediatr Res. 2016;79(1-2):191-6.

20. Friedman SL, Kalichman MA, Council on Children with D. Out-of-home placement for children and adolescents with disabilities. Pediatrics. 2014;134(4):836-46.

21. O'Brien JE, Berry J, Dumas H. Pediatric post-acute hospital care: striving for identity and value. Hosp Pediatr. 2015;5(10):548-51.

22.• Berry JG, Hall M, Dumas H, et al. Pediatric hospital discharges to home health and postacute facility care: a national study. JAMA Pediatr. 2016;170(4):326-33.

This recent publication brings our attention to an underutilized approach to the care of CMC that might contribute to lower costs and better outcomes for $\mathrm{CMC}$ and their families.

23. Smith AG, Andrews S, Bratton SL, et al. Pediatric palliative care and inpatient hospital costs: a longitudinal cohort study. Pediatrics. 2015;135(4):694-700.
24. Ralston SL, Harrison W, Wasserman J, Goodman DC. Hospital variation in health care utilization by children with medical complexity. Pediatrics.

2015;136(5):860-7.

25. Stenger MR, Slaughter JL, Kelleher K, et al. Hospital variation in nitric oxide use for premature infants. Pediatrics. 2012;129(4):e945-51.

26. Thomson J, Hall M, Ambroggio L, Stone B, Srivastava R, Shah SS, et al. Aspiration and NonAspiration Pneumonia in Hospitalized Children With Neurologic Impairment. Pediatrics. 2016;137(2):e20151612.

27. Van Cleave J, Okumura MJ, Swigonski N, O'Connor KG, Mann M, Lail JL. Medical Homes for Children With Special Health Care Needs: Primary Care or Subspecialty Service? Acad Pediatr. 2016;16(4):366-72.

28. Gordon JB, Colby HH, Bartelt T, Jablonski D, Krauthoefer ML, Havens P. A tertiary care-primary care partnership model for medically complex and fragile children and youth with special health care needs. Arch Pediatr Adolesc Med.

2007;161(10):937-44.

29. Casey PH, Lyle RE, Bird TM, et al. Effect of hospital-based comprehensive care clinic on health costs for Medicaid-insured medically complex children. Arch Pediatr Adolesc Med.

2011;165(5):392-8

$30 . \bullet \quad$ Mosquera RA, Avritscher EB, Samuels CL, et al. Effect of an enhanced medical home on serious illness and cost of care among high-risk children with chronic illness: a randomized clinical trial. JAMA. 2014;312(24):26408 .

This is the first randomized-controlled trial to assess the impact of comprehensive care for CMC.

31. Kuo DZ, Berry JG, Glader L, Morin MJ, Johaningsmeir S, Gordon J. Health services and health care needs fulfilled by structured clinical programs for children with medical complexity. J Pediatr. 2016;169:291-6. e291.

32. Bekmezian A, Chung PJ, Yazdani S. Staff-only pediatric hospitalist care of patients with medically complex subspecialty conditions in a major teaching hospital. Arch Pediatr Adolesc Med. 2008;162(10):975-80.

33. Bogetz JF, Bogetz AL, Rassbach CE, Gabhart JM, Blankenburg RL. Caring for children with medical complexity: challenges and educational opportunities identified by pediatric residents. Acad Pediatr. 2015;15(6):621-5.

This paper identifies gaps in current pediatric residency experiences and describes opportunities to promote a future pediatric workforce that will be better prepared to address the unique healthcare needs of $\mathrm{CMC}$. 